\title{
An Exfoliative Cytological Study of Three Cases of Oral Sarcoma
}

\author{
by \\ Naoto KYODA, Shinkichi IWANARI, Yuji DAITO, \\ Itsuro KUDO and Takao WATANABE*
}

Introduction

Compared with carcinomas, the incidence rate of oral sarcoma is extremely low[1]. There are a relatively small number of histological studies on these sarcomas, but even fewer cytological ones[2,3,4]. In 1980 we treated 3 cases of sarcoma and used that opportunity to gather cytological information about oral sarcomas. Specifically we 1) tried to identify the general cytological characteristics of oral sarcomas, 2) compared cytological findings with previous histological research[5], showing that oral sarcomas are morphologically different from sarcomas in other regions, and 3 ) examined the possibility of identifying various sarcoma types using cytological techniques.

\section{Case Report}

Case 1: K.T., a 31-year-old male (fibrosarcoma)

In August 1980, after noticing a painless swelling in the lower gingiva at the incisors, the patient went to a dentist and the right lower incisors were extracted. However, that area continued to increase in size and gradually became painful. On his dentist's recommendation he visited Nihon University Dental Hospital in October 1980. His general physical condition was good and no facial irregularities were visible. However, the submandibular lymph nodes on the right were the size of an adzuki bean, movable and hard, while those on the left were pea-sized, rigid, hard and slightly painful when pressed. Also there was a tumor about $2.5 \mathrm{~cm}$ in diameter in the gingiva around the lower incisors. It was soft, slightly red, bled easily, had a rough surface and it was partly ulcerated. The lower left first and second incisors were missing. A panoramic radiograph (Fig. 1) revealed a poorly delimited radiolucent zone and the absence of the margin of alveolar bone in the lower jaw from the first lower right incisor to the lower left canine. Other clinical examinations showed results within normal limits, except for an acceleration of the erythrocyte sedimentation rate.

In December 1980, we performed a segmental mandibulectomy to extract the tumor. As of yet, no metastasis or local recurrence has been found.

京田直人, 岩成進吉, 大塔雄二, 工藤逸郎：Dept. of Oral Surgery, Nihon University School of Dentistry, Tokyo, Japan. (Director: Prof. Itsuro KUDO)

*渡辺孝夫: The First Dept. of Oral Surgery, Tsurumi University School of Dental Medicine, Yokohama, Japan. (Director: Prof. Kan-ichi SETO) 
Cytological Findings (Figs. 2 and 3):

Many spindle, fibrous and round cells were found scattered throughout the slides. A few other cells had bizarre shapes. The cytoplasm of most cells displayed a fine net-like structure and stained light green with Papanicolaou stain. Round or oval nuclei with unusually thin nuclear membranes, irregular thickening of nuclear membranes, granular chromatin, hyperchromatism and lobulation were observed. The nuclei and nucleoli showed a tendency to increase in number and size. Anisocytosis and anisokariosis were prominent. Some polynuclear giant cells had several small nuclei, as if they were a result of splitting. Also, inflammatory cells were found.

Histological Findings (Fig. 4):

The tumor was composed mostly of spindle cells, some of which were in mitosis, showing irregular nuclei, hyper chromatism and prominent nucleoli. In some areas, collagenous fiber bundles arranged in a herringbone pattern were seen. Also some large, poorly differentiated spindle cells with plump nuclei were observed.

Case 2: N.M., a 17-year-old female (fibrosarcoma)

In July 1980, during routine treatment for several cavities, a poorly delimited swelling on the right side of the patient's lower jaw was found. Her dentist then recommended that she visit our hospital for a more thorough examination.

Her first visit was in September, 1980. Her overall physical condition was good and her face appeared slightly asymmetrical. This was found to be due to a hard, painless diffuse swelling on the lower right part of her jaw. The right submandibular lymph nodes were the size of a pea, movable and soft. Inside her mouth there was an approximately $2 \mathrm{~cm}$ hemispherical swelling in the lower right jaw at the molars, which was as hard as bone tissue, painless and not clearly delimited. Neither redness nor ulceration was present. The first and second molars were vital, movable and painless when pressed. A panoramic radiograph (Fig. 5) revealed a poorly delimited radiolucent region in the lower right jaw extending from the second premolar to the third molar. Clinical examinations showed results within normal limits except for an acceleration of the erythrocyte sedimentation rate.

In October 1980, in order to extract the tumor, a segmental mandibulectomy was performed at another hospital. No metastasis or local recurrence has yet been found.

Cytological Fingings (Figs. 6 and 7):

Most cells observed from this case were spindly, similar to those found in Case 1 and cells in mitosis were clearly observable.

Histological Findings (Fig. 8):

Abundant fibrous bundles, with spindle and fibrous cells, were arranged in a herringbone pattern. The nuclei varied in shape and size. Irregularities, as in the shape of nuclei, hyperchromatism and enlargement of nucleoli were observed. Cells in mitosis were numerous.

Case 3: Y.M., a 38-year-old female (chondrosarcoma)

In April 1980, the patient noticed a swelling in the gingiva at the lower left molars. Although it was painless, it continued to increase in size, so she eventually 


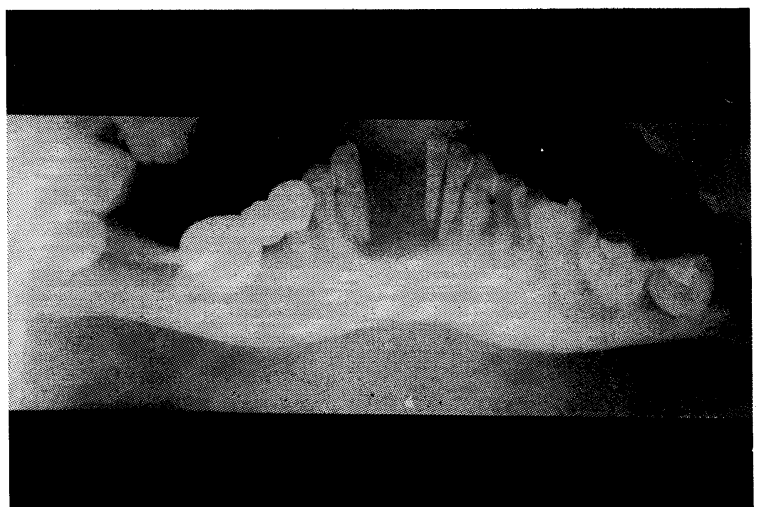

Fig. 1 (Case 1) A panoramic radiograph revealed a poorly delimited radiolucent zone in the incisor region of the lower jaw.

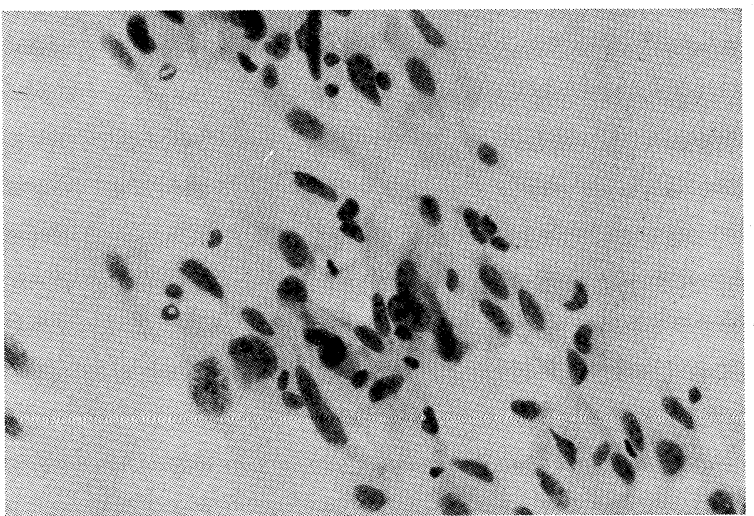

Fig. 2 (Case 1) Low magnification of a cytological specimen. Many spindle cells are seen. Some cells have large nuclei with coarse granular chromatin (Pap. stain).

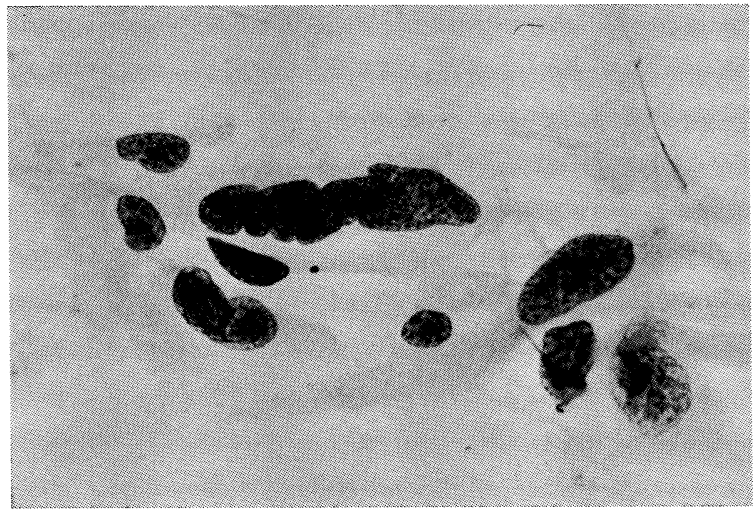

Fig. 3 (Case 1) High magnification of a cytological specimen. Irregularities of nuclear contour are prominent (Pap. stain). 


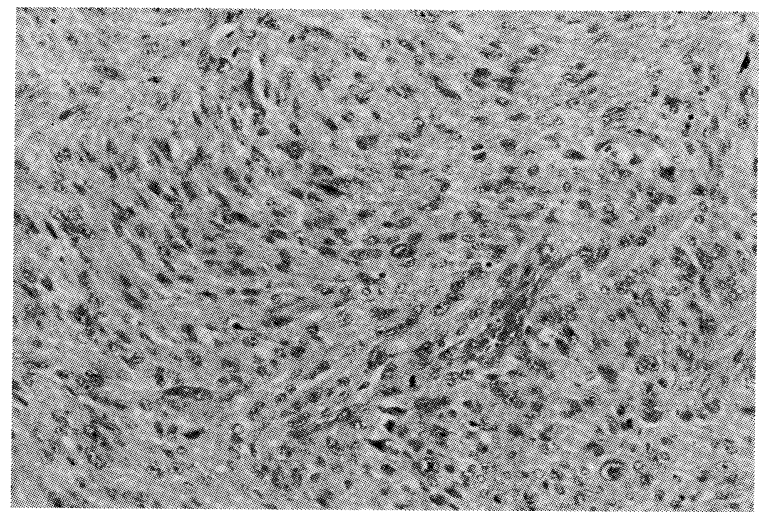

Fig. 4 (Case 1) Tumor composed of spindle cells. Cells in mitosis are present (H-E stain).

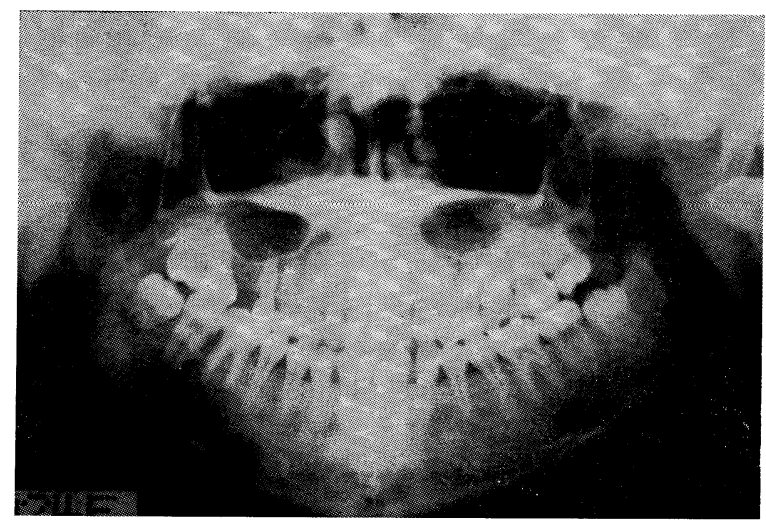

Fig. 5 (Case 2) A panoramic radiograph revealed a poorly delimited radiolucent zone in the molar region of the lower right jaw.

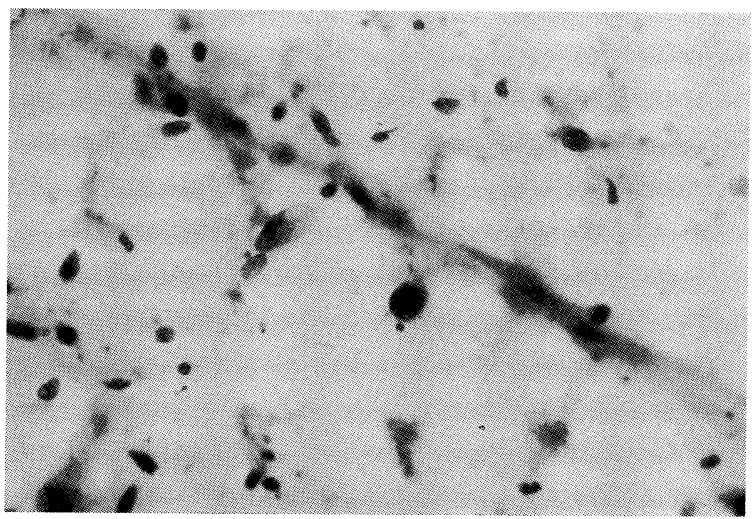

Fig. 6 (Case 2) Low magnification of a cytological specimen. Small spindle cells which indicate slight anisocytosis are scattered (Pap. stain). 


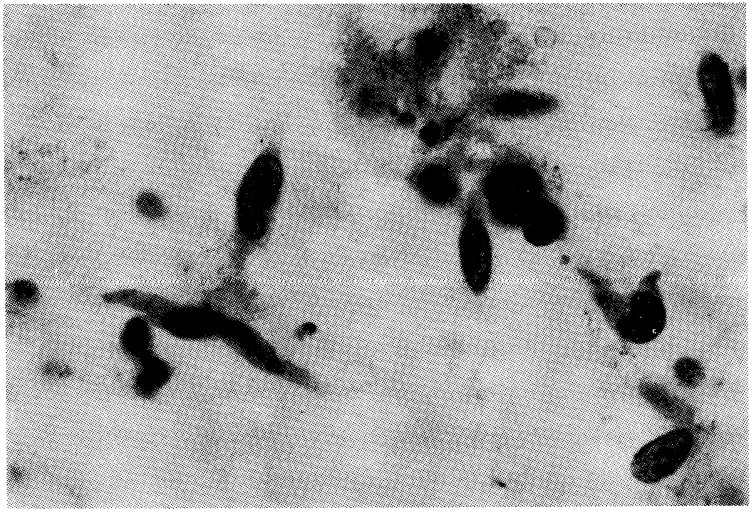

Fig. 7 (Case 2) High magnification of a cytological specimen. Spindle cells with nuclei included in abundant chromatin are observed (Pap. stain).

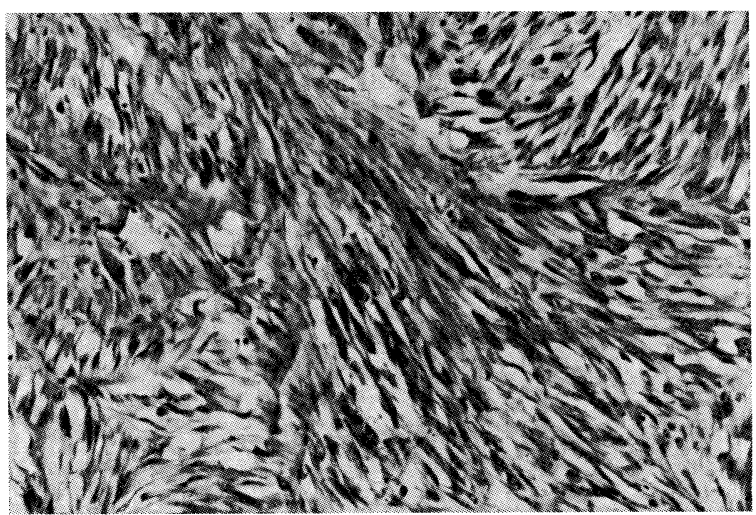

Fig. 8 (Case 2) Spindle cells are predominent. They form bundles like a herringbone pattern (H-E stain).

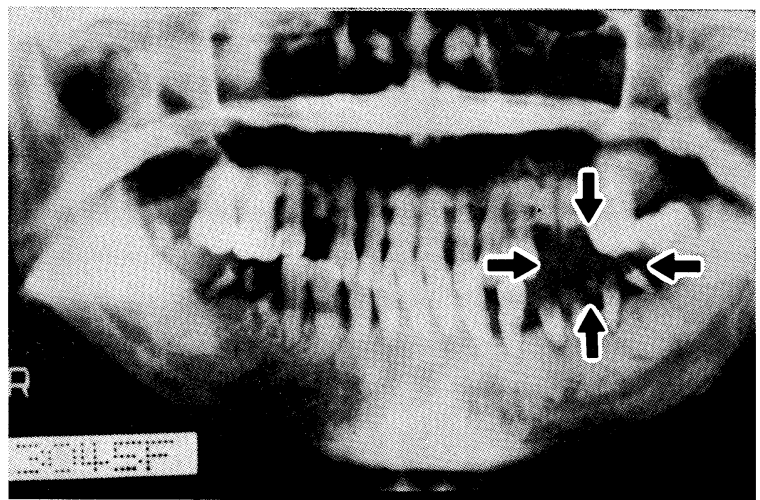

Fig. 9 (Case 3) Radiolucent tumor is seen in the gingiva of the lower left jaw (arrows). It contains several radioopaque structures. 


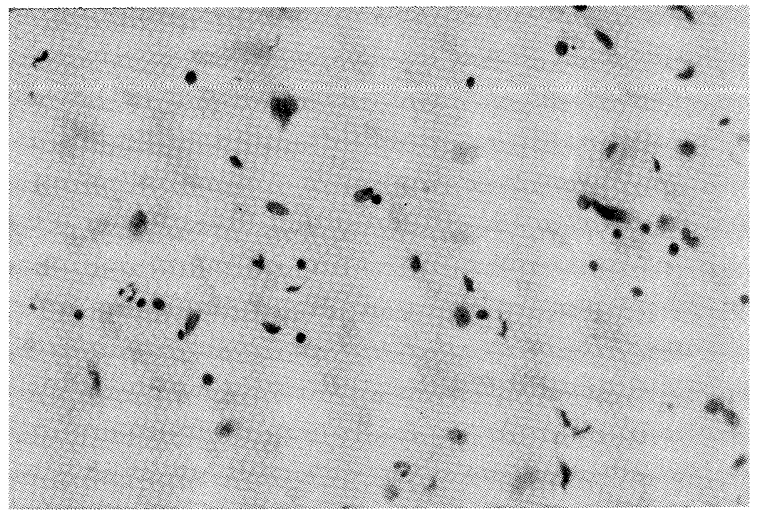

Fig. 10 (Case 3) Low magnification of a cytological specimen. Small spindle and round cells are scattered. A cell has a few nuclei (Pap. stain).

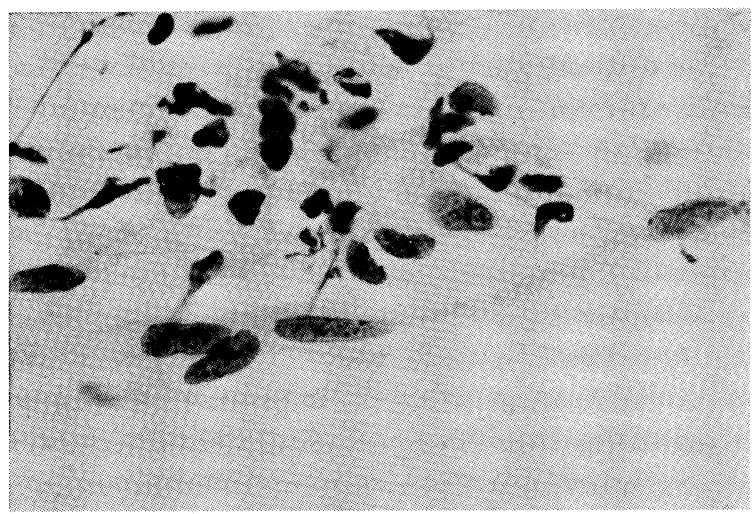

Fig. 11 (Case 3) High magnification of a cytological specimen. Several fibrous cells with ovoid nuclei are seen. Note the uneven nuclear membrane in some cells (Pap. stain).

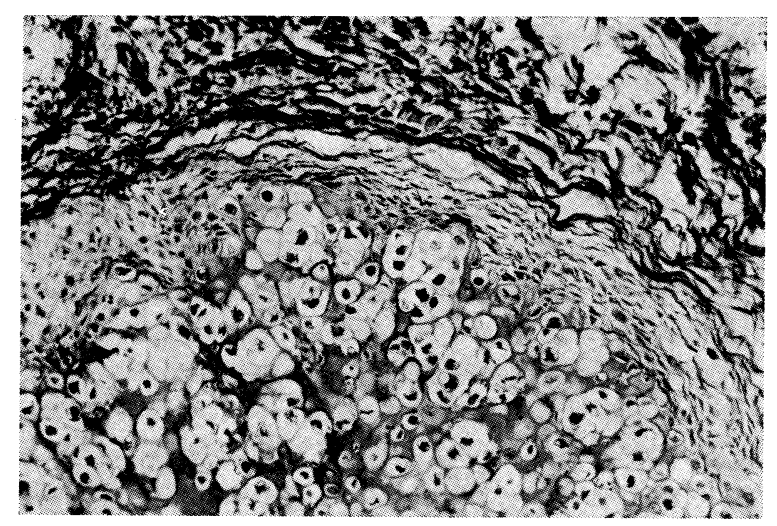

Fig. 12 (Case 3) Spindle cells with plump nuclei are seen in the periphery of chondroid substances (H-E stain). 
went to a dentist. He suggested that she go to our hospital.

Her first visit was in July 1980. At that time, her face appeared slightly asymmetrical because of the swelling in her lower jaw. Two of the right submandibular lymph nodes were palpable and movable, while only one was painful when pressed. Also there was a tumor in the gingiva extending from the left second premolar to the left second molar, which was the size of a hen's egg, hard and painless, and had irregular contours. A panoramic radiograph (Fig. 9) revealed an exophytic tumor of the lower left jaw which was radiolicent with numerous irregularly shaped, slightly opaque regions. Under the lesion, the upper part of the alveolar bone had disintegrated, but the cortex was intact. The results of clinical examinations were within normal limits.

In September 1980 the tumor was extracted by means of a marginal mandibulectomy. As of yet, no metastasis or local recurrence has occurred.

Cytological Findings (Figs. 10 and 11)

Spindle cells with round or oval nuclei were abundant and the nuclei varied in size. Nuclear irregularities, such as lobulation, irregular thickening of the nuclear membrane, and hyperchromatism were prominent. However, multinucleated giant cells were less numerous than in Cases 1 and 2.

Histological Findings (Fig. 12)

Most parts of the tumor showed many nests composed of spindle and oval cells with plump nuclei and abundant cytoplasm. In some parts, collagenous fiber bundles were present. Chondroid substances were seen in central parts of the nests. Portions of the chondroid substances were calcified. Many mononuclear and binucleate malignant chondrocytes, varying in size and shape, were impacted in the chondroid substances. Some areas showed a high concentration of cells. In other areas, myxomatous tissue was seen. Cells in mitosis were also observed.

\section{Discussion}

Cell Grouping, Cytoplasm (Table 1a):

In all the cases as well as the control (an apical granuloma consisting mainly of normal fibroblasts and fiber cells), the cytoplasm was cyanophilic and appeared lacy. These findings may be characteristic of mesenchymal tamor calls $[6,7,8,9]$. "Rosary clusters[6]" (single cells attached to each other in a chain-like formation) which are often seen in leiomyosarcoma and abdomyosarcoma but not in fibrosarcoma were not present. In distinguishing the cells by contour the following classifications were used; spindly, fibrous, round, and others. The results revealed that in the two fibrosarcomas and the control, spindle cells were the most numsrous $(23-29 \%)$, followed by round-type cells $(18-20 \%)$.

The ratio of each type of cell to total cells observed in the two fibrosarcomas and the control were very similr to each other. However, fibrous cells were predominant in the chondrosarcoma. This differs from HADJU's[10] and others' findings that the most numerous cells were round. Perhaps, by chance our samples happened to be from an area with an unusually high concentration of fibrous cells, and were not representative of the whole. Or perhaps fibrous cells are indeed more numerous in chondrosarcomas than was previously reported by other researchers. In any 
Table 1a Cell Grouping and Cytoplasm* (Pap. stain)

\begin{tabular}{|c|c|c|c|c|}
\hline & Case 1 & Case 2 & Case 3 & \\
\hline & Fibrosarcoma & Fibrosarcoma & Chondrosarcoma & $\begin{array}{l}\text { Radicular } \\
\text { granuloma** }\end{array}$ \\
\hline Cell grouping & loose & loose & loose & loose \\
\hline Color & blue & blue & blue & blue \\
\hline Staining property & pale & pale & pale & pale \\
\hline Cell shape: spindly & $43 \%$ & $44 \%$ & $17 \%$ & $47 \%$ \\
\hline fibrous & $23 \%$ & $23 \%$ & $63 \%$ & $29 \%$ \\
\hline round & $19 \%$ & $20 \%$ & $3 \%$ & $18 \%$ \\
\hline others & $15 \%$ & $13 \%$ & $17 \%$ & $6 \%$ \\
\hline Coalescent boundary & $72 \%$ & $81 \%$ & $52 \%$ & $100 \%$ \\
\hline Structure & lacy & lacy & lacy & lacy \\
\hline
\end{tabular}

Table 1b Nucleus and Nucleolus* (Pap. stain)

\begin{tabular}{ccccc}
\hline & Case 1 & Case 2 & Case 3 & $\begin{array}{c}\text { Radicular } \\
\text { granuloma** }\end{array}$ \\
\hline Nuclear form: oval & $55 \%$ & $75 \%$ & $74 \%$ & $71 \%$ \\
round & $43 \%$ & $20 \%$ & $25 \%$ & $24 \%$ \\
others & $2 \%$ & $5 \%$ & $1 \%$ & $5 \%$ \\
Uneven nuclear contour & $49 \%$ & $42 \%$ & $47 \%$ & - \\
Chromatin: coarse & $26 \%$ & $58 \%$ & $54 \%$ & - \\
fine & $69 \%$ & $41 \%$ & $46 \%$ & - \\
Irregular nuclear membrane & $59 \%$ & $39 \%$ & $57 \%$ & $100 \%$ \\
Number of nuclei: 1 & $89 \%$ & $83 \%$ & $98 \%$ & - \\
2 & $8 \%$ & $12 \%$ & $2 \%$ & - \\
3 or more & $3 \%$ & $5 \%$ & - & $12 \%$ \\
Number of nucleoli:0 & $13 \%$ & $7 \%$ & $12 \%$ & - \\
1 & $39 \%$ & $25 \%$ & $40 \%$ & - \\
2 or more & $48 \%$ & $68 \%$ & $48 \%$ & \\
\hline
\end{tabular}

Table 1c Cell and Nuclear Size, and Nucleo-Cytoplasmic Ratio (Pap. stain)

\begin{tabular}{crrrr}
\hline & Case 1 & Case 2 & Case 3 & $\begin{array}{l}\text { Radicular } \\
\text { granuloma** }\end{array}$ \\
\hline Cell long axis: Max. & $152.5 \mu$ & $90.0 \mu$ & $110.0 \mu$ & $40.0 \mu$ \\
Min. & $6.3 \mu$ & $8.8 \mu$ & $8.8 \mu$ & $15.0 \mu$ \\
Av. & $32.4 \mu$ & $28.7 \mu$ & $33.8 \mu$ & $32.15 \mu$ \\
short axis: Max. & $38.8 \mu$ & $35.0 \mu$ & $16.3 \mu$ & $13.0 \mu$ \\
Min. & $3.8 \mu$ & $5.0 \mu$ & $5.0 \mu$ & $5.0 \mu$ \\
Av. & $9.2 \mu$ & $8.4 \mu$ & $9.6 \mu$ & $8.69 \mu$ \\
Nucleus long axis: Max. & $21.3 \mu$ & $36.3 \mu$ & $17.5 \mu$ & $15.0 \mu$ \\
Min. & $5.0 \mu$ & $5.0 \mu$ & $5.0 \mu$ & $6.0 \mu$ \\
Av. & $10.9 \mu$ & $12.6 \mu$ & $10.9 \mu$ & $11.6 \mu$ \\
short axis: Max. & $13.8 \mu$ & $11.3 \mu$ & $7.5 \mu$ & $10.0 \mu$ \\
Min & $2.5 \mu$ & $3.8 \mu$ & $3.8 \mu$ & $4.0 \mu$ \\
Av. & $7.0 \mu$ & $6.1 \mu$ & $5.5 \mu$ & $7.0 \mu$ \\
N/C ratio: long axis Av. & 0.3364 & 0.4390 & 0.3225 & 0.3612 \\
short axis Av. & 0.7609 & 0.7262 & 0.5729 & 0.8053 \\
\hline
\end{tabular}

*number per 100 randomly selected tumor cells

**only fibroblasts and fiber cells studied 
case, it is extremely difficult to determine histological classifications just from cell type.

Although the ratio of cells with indistinct cellular membranes was over $70 \%$ for both fibrosarcomas and the control, it was about 50\% for the chondrosarcoma. Previous reports on the state of the cell wall after staining vary. Some researchers observed that the cell walls tend to remain intact[6], while others found that they tend to disintegrate[11,12]. This discrepancy may be caused by differences in their individual techniques for obtaining and staining materials. Yet few researchers have provided information on the actual ratio of cells with intact membranes.

The cytoplasm in our samples displayed a net-like or lacy pattern, which corresponds to the findings of other researchers[13,14]. Some cells in all cases, except the control, had foreign bodies in the cytoplasm. Few researchers[14,15] have mentioned whether or not foreign bodies are present in cells from nonepithelial tumors.

Nucleus and Nucleolus (Table 1b):

According to previous literature, most nonepithelial malignant tumors are composed of cells having primarily round or oval-shaped nuclei[ $[9,12,13,16]$. The shape of the nucleus in benign nonepithelial lesions shows a similar tendency[17]. Yet we wondered if percentages of cells with the different shaped nuclei may provide a clue for distinguishing between malignant and benign lesions. The data show no significant variation and it would appear that differences in nuclear shape cannot be used for diagnostic purposes.

Uneven nuclear contours appeared in $42-44 \%$ of the tumor calls in the sarcoma cases. However, no such irregularities were found in cells from the control. Naclear membrane irregularities, such as depression or lobulation, in DFSP[16], SCF[16], leiomyosarcoma[6] and MFH[16] have been suggested as characteristic of malignant lesions.

Although KaKiHANa[16] observed cells with thickened, irregularly shaped nuclear membranes in SCFS, it is commonly thought that the nuclear membrane in nonepithelial tumor cells is thin[6,8,9,12]. Our results showed thickened membranes accompanied by irregularities in $39-59 \%$ of the cells from the sarcoma cases, but no such thickening was seen in the control. This indicates that thickening and irregularity of the nuclear membrane may be of significance in the diagnosis of nonepithelial as well as epithelial lesions.

Two classifications were used for the appearance of the chromatin; fine-granular and coarse-granular. In Case 1, the percentage of cells with coarse-granular chromatin was $26 \%$, but $58 \%$ for Case 2 and $54 \%$ for Case 3 . In the control, coarsegranular chromatin was observed in $26 \%$ of the tumor cells counted. Although there are few reports indicating statistically the proportion of cells displaying the two types of chromatin, most researchers[18] have stated that cells from nonepithelial lesions tend to have fine-granular chromatin. Some authors[7,12], however, have observed coarse-granular chromatin in a few leiomyosarcoma cells. As the present study revealed a relatively high ratio of cells with coarse-granular chromatin only in the sarcoma cases, it would seem that the aspect of the chromatin can be another factor to consider in diagnosing cell malignancies.

A tendency to have an increased number of nuclei was observed in the sarcoma 
Table 2 Other Stains

\begin{tabular}{lcccccc}
\hline & \multicolumn{2}{c}{ Case 1} & & \multicolumn{2}{c}{ Mvxoma } \\
\cline { 2 - 3 } & Cell body & Background & & Cell body & Background \\
\hline PAS & - & - & & - & + \\
Mucicarmin & - & - & & - & + \\
Alcian blue & - & - & & - & + \\
AZAN Mallory & - & & - & & - & - \\
Elastica van GIESON & - & - & - & & - & - \\
PTAH & - & - & & - & - \\
Silver & - & + & & - & + \\
\hline
\end{tabular}

cases, while most cells in the control were mononuclear. These results were identical with those of other researchers[6,7,14]. Multinucleated cells have also generally been seen in non-malignant lesions[16], such as chronic inflammation and benign tumors. However, the multinucleated cells found in this study also showed irregularly shaped nuclei and variations in nuclear size-alterations that do not appear to accompany chronic inflammation or benign tumors.

As to the number of nucleoli, the percentage of cells with two or more nucleoli was $48 \%$ in Cases 1 and 3, 68\% in Case 2, while in the control, nucleoli were either absent or singular in number. So, it is apparent that there is a tendency toward multiple nucleoli in sarcoma cases. In previous research, there are two contradictory views that the nucleoli are small and not predominent[10,12,16,19] and that they are prominent and numerous[6,7,9,19], but our results suggest that the presence and number of nucleoli is important in cytological diagnosis.

Axial Measurements and Nucleo-Cytoplasmic Ratio (Table 1c)

Measurements of the long and short axes of cells and nuclei are recorded in Table 1c. From the data it is apparent that the difference between the maximum and the minimum length of the cells' long axis in the sarcoma cells is greater than that in the control. As to the short axis, the difference was larger in Cases 1 and 2 than in Case 3 and the control. The average lengths of the long and short axes were similar in all cases as well as in the control. While the measurements recorded for the long and short nuclear axes show only a slight difference in maximum, minimum, and average length from case to case, the sarcoma cells tended to have larger nuclei and show a wider range of axial measurements. Anisocytosis and anisokariosis are thought to be among the significant identifying characteristics of malignant cells not only in epithelial tumors but also in nonepithelial tumors[6,7,8,9,10,16,20], and our results showed a similar trend.

The nucleo-cytoplasmic ratio (N/C) of the long and short axes was calculated in order to further compare the cases. The results revealed no significant differences between the sarcomas and the control, in spite of the observation that tumor cells with comparatively high $\mathrm{N} / \mathrm{C}$ ratios were sometimes seen in the sarcomas.

Staining (Table 2):

To examine the utility of histochemical staining, we performed PAS, mucicarmine, alcian blue, AZAN Mallory, elastica van GIESON, PTAH and silver 
staining on cells from Case 1. As a result, dark green granules staincd by AZAN Mallory were present in the cytoplasm of some cells and a few fine black fibers (positive response to silver stain) were observed in areas outside of the tumor cells themselves. Except for these results, the various staining techniques were negative.

\section{Conclusion}

In this study, we have tried to examine the cytological characteristics of three sarcomas taken from patients whom we recently treated. The results showed that irregularity of the nuclear membrane, number of nuclei, thickening of the nuclear membrane, aspect of chromatin, number of nucleoli, cellular and nuclear size, anisocytosis and anisokaryosis may be significant characteristics in determining malignancy in sarcomas through cytological methods. In response to the three points raised in the introduction, we found that: 1) the cytological characteristics observed seemed similar to findings of previous histological and cytological research, 2) there did not seem to be any striking cytological differences between oral sarcomas and sarcomas in other regions and 3) we were unable to distinguish clearly between various oral sarcoma types on the basis of cytological studies alone.

\section{Acknowledgment}

We would like to thank Prof. Yoshio Watanabe and Prof. Kan-ichi Seto, Tsurumi University School of Dental Medicine, for reading a draft of the manuscript and making helpful suggestions and Mrs. Margaret E. OLIN for her advice in the translation.

\section{References}

[1] UEno, T. et al. 1961. Incidence of oral malignant tumors. Bull. Tokyo Med. Dent. Univ. 8, 7181.

[2] Watanabe, T. et al. 1980. Two cases of malignant non-epithelial tumors in the maxilla. $J$. Jpn. Soc. Clin. Cytol. 19, 355.

[3] Watanabe, Y. 1964. Exfoliative cytology of maxillo-oral sarcoma. XII. Ann. Scientific Mtg. Amer. Soc. Cytology, Pittsburgh.

[4] Watanabe, T. et al. 1982. A case of juvenile ossifying fibroma in the mandible. J. Jpn. Soc. Clin. Cytol. 21, 415.

[5] Ushigome, S. 1979. Sarcomas of the jaw-differential diagnosis of osteosarcoma, fibrosarcoma and malignant fibrous histiocytoma-. J. St. M. Univ. Sch. Med. 7, 399-410.

[6] Yamada, Y. 1974. Cytology of non-epithelial tumors. J. Jpn. Soc. Clin. Cytol. 13, 139-155.

[7] Katow, K. 1974. Cytological study of the sarcoma in the uterine cervix. J. Jpn. Soc. Clin. Cytol. 13, 55-58.

[8] MukaI, H. 1978. Cytology of non-epithelial malignant tumors. J. Jpn. Soc. Clin. Cytol. 17, 461.

[9] Takahashi, M. 1978. Cytologic study of sarcoma and carcinosarcoma in uterus. J. Jpn. Soc. Clin. Cytol. 17, 495.

[10] Hajdu, S. I. and Hajdu, E. D. 1976. Cytopathology of sarcomas and other non-epithelial malignant tumors. W. B. Saunders, Toronto.

[11] Tanaka, T. 1977. A case of osteosarcoma diagnosed cytologically. J. Jpn. Soc. Clin. Cytol. 16, 109-112.

[12] SAwADA, K. 1977. Cytological diagnosis of metastatic lung tumors, with special reference to transthoracic needle biopsy. J. Jpn. Soc. Clin. Cytol. 16, 286-293.

[13] Hukushima, N. 1975. A case of paraosteal oseosarcoma in lung-cytological study with needle 
biopsy. J. Jpn. Soc. Clin. Cytol. 14, 270.

[14] Takeda, K. 1979. Cytological studies of osteogenic sarcomas. J. Jpn. Soc. Clin. Cytol. 18, 391.

[15] Yamada, T. 1979. Differential cytodiagnosis of sarcoma cells by means of various stains. $J$. Jpn. Soc. Clin. Cytol. 18, 336-337.

[16] Kakihana, M. 1974. Exfoliative cytology of malignant neoplasmas of the non-epithelial origin - cytologic morphology of malignant fibrous tumors. J. Jpn. Soc. Clin. Cytol. 13, 59-67.

[17] Sanerkin, N. G. 1980. Cytology of bone tumours. John Wright \& Sons Ltd., Bristol.

[18] Sakuma, Y. 1979. Cytological study of two cases of osteogenic malignant fibrous histiocytoma. J. Jpn. Soc. Clin. Cytol. 18, 212.

[19] UedA, S. 1977. Exfoliative cytology of a malignant fibrous histiocytoma arising in a dermoid cyst of the ovary. J. Jpn. Soc. Clin. Cytol. 16, 282-285.

[20] Kobayashi, Y. 1971. Cytology of some kinds of non-epithelial tumors. J. Jpn. Soc. Clin. Cytol. 10, 308. 